\title{
Einleitung: das Universum von Möglichkeiten
}

\author{
„Die ökonomische und soziale Welt - Positionen, die \\ man einnehmen, \\ Bildungswege, die man einschlagen, Güter, die man \\ konsumieren, Besitztümer, \\ die man kaufen, und Frauen, die man heiraten kann, \\ usw. - nimmt niemals, es sei denn \\ in der Einbildung, bei außer Kraft \\ gesetztem Realitätssinn, die Gestalt eines Universums \\ von Möglichkeiten an, die jedem beliebigen Subjekt \\ gleichermaßen offenstehen.“
}

(Bourdieu 1981: 180)

$79 \%$ der Kinder in Deutschland, die mindestens einen Elternteil mit Hochschulabschluss haben, nehmen ein Studium auf - unter den Kindern aus Familien ohne Hochschulerfahrung sind es nur $27 \%$ (Kracke/Buck/Middendorff 2018: 4). Gleichzeitig beginnt nur jede zehnte Frau, die sich an einer deutschen Hochschule immatrikuliert, ein ingenieurwissenschaftliches Studium. Umgekehrt entscheidet sich jeder zehnte Student für ein pädagogisches oder sozialwissenschaftliches Studienfach (Middendorff et al. 2017: 14). Kurzum: Wer überhaupt studiert und welches Studienfach dafür in Frage kommt wird sowohl von der sozialen Herkunft wie auch dem sozialen Geschlecht maßgeblich bestimmt. Die ,ökonomische und soziale Welt" mag vor dem Hintergrund von Bildungs- und Berufswegen grundsätzlich jedem Subjekt offenstehen, die damit verbundenen sozialen Positionen allerdings nicht in gleichem Maße. 
Das einleitende Zitat von Pierre Bourdieu weist in mehrerer Hinsicht auf die zentralen Anliegen dieser Arbeit hin: Ziel ist zum einen, das „Universum von Möglichkeiten“ und seine einschränkenden Mechanismen der kulturellen Passung im Bereich der Studien(fach)- ${ }^{1}$ und Berufswahl offenzulegen - also die sozialen Logiken zu entschlüsseln, die am Übergang in die Hochschule zur Reproduktion sozialer Ungleichheit beitragen. Zum anderen möchte diese Arbeit einen Beitrag dazu leisten, einen geschlechtssensiblen Blick in der Bildungsforschung im Anschluss an Bourdieu anzuwenden. Dieser Blick fehle im Bourdieuschen Ansatz, folgt man einem dominanten Rezeptionsmuster seiner Arbeit. So schlagen sich auch im obigen Zitat Androzentrik und Heteronormativität augenscheinlich nieder - schließlich wird das dortige Subjekt als per se männliches gesetzt und Frauen als davon abhängige konsumgutartige Objekte. Allerdings haben Teile der Geschlechterforschung das geschlechtssensible Potential Bourdieus erkannt und nutzen seinen Zugang zur theoretischen wie empirischen Betrachtung von Ungleichheiten.

Dass das Geschlecht als zentrale Dimension sozialer Ungleichheit immer mitgedacht und empirisch berücksichtigt werden muss, wird allgemein in der Bildungsforschung mittlerweile vorausgesetzt. Nichtsdestotrotz gehen viele Studien nur so weit, Geschlecht als binäres Verteilungsverhältnis zu betrachten, ohne die Prozesse seiner Herstellung in den Blick zu nehmen. Umgekehrt vermag die Geschlechterforschung zwar, Ausschluss- und Abdrängungsmechanismen in Studien(fach)wahlprozessen qua Geschlecht pointiert herauszuarbeiten, vernachlässigt aber die Bedeutung der sozialen Herkunft. Die theoretischen und empirischen Perspektiven von Geschlechter- und Bildungsforschung miteinander zu verweben und auf dieser Grundlage das Zusammenspiel von sozialer Herkunft und Geschlecht in Studien(fach)wahlen zu analysieren, sind die Ziele dieser Arbeit.

\section{Studien(fach)wahlen in der Geschlechterforschung}

Studien(fach)wahlen liegen im besonderen Interesse der Geschlechterforschung, da sie als Schritt in ein spezifisches Berufsfeld sowohl zur Geschlechtersegregation in den Studiengängen als auch perspektivisch in den entsprechenden Berufsfeldern führen. In geschlechtersoziologischen Betrachtungen steht deshalb weniger die Frage danach im Vordergrund, ob Frauen und Männer überhaupt ein

\footnotetext{
${ }^{1}$ Der Begriff der ,Studien(fach)wahl ‘ umfasst sowohl die grundsätzliche Entscheidung für ein Studium wie auch jene für ein spezielles Studienfach. Ist im Folgenden dagegen von ,Studienfachwahl` die Rede, meint das ausschließlich die fachliche Dimension.
} 
Studium aufnehmen - denn dieses Verhältnis ist in etwa ausgeglichen - sondern vielmehr die ungleiche Verteilung der Geschlechter auf die verschiedenen Fächer. In diesem Sinne ist die Studien(fach)wahl ein zentraler Mechanismus in der fachlichen Segmentierung des Arbeitsmarktes: Bestimmte Berufe werden überwiegend von Frauen, andere überwiegend von Männern ausgeübt. Damit trägt die Studien(fach)wahl auch zur geschlechtlichen Arbeitsteilung bei, die neben der fachlichen Segmentierung des Arbeitsmarktes zweitens die vertikale Segregation von Berufen - also die ungleiche Verteilung von Frauen und Männer auf die verschiedenen beruflichen Hierarchiestufen - und drittens die überwiegende Ausübung unbezahlter Reproduktionsarbeit durch Frauen umfasst. ${ }^{2}$ In der Konsequenz sind Frauen häufiger in schlechter vergüteten Dienstleistungsberufen und in den Feldern Erziehung und Pflege vertreten, sie führen mehr Familienarbeit aus etwa in Form von Erziehung und Pflege von Angehörigen - und sie nehmen (auch in weiblich dominierten Feldern ${ }^{3}$ ) die rangniedrigeren und sozial wie finanziell weniger anerkannten Positionen ein. Analysen der berufsbezogenen Geschlechterforschung zielen darauf $a b$, die Bedeutung der Geschlechtszugehörigkeit für die Strukturierung und/oder die Symbolisierung von Berufsarbeit herauszuarbeiten (Teubner 2008: 491).

Dabei interessieren aktuelle Ansätze der Geschlechterforschung vor allem die Ursachen für den geringen Frauenanteil in den Fächern Mathematik, Informatik, Naturwissenschaft und Technik - dem sogenannten MINT-Bereich - und die daran anschließenden Möglichkeiten, diesen Anteil zu erhöhen. Weshalb Männer seltener ein soziales Studium beginnen, liegt zwar auch im Erkenntnisinteresse der Geschlechterforschung, wird allerdings seltener verfolgt als die Frage nach dem Fehlen von Frauen in Ingenieurfächern. Denn der Ruf nach Frauen in MINT ist ein qualitativ anderer als der nach Männern in sozialen Berufen: Bei dem einen steht die Frage nach Chancengleichheit und der Zugang zu den statusund zukunftsträchtigen technisch-naturwissenschaftlichen Berufsfeldern im Vordergrund, während bei dem anderen die Intention eine pädagogische ist mit dem Ziel einer vermeintlich ,binären Vollständigkeit“ (May/Rose 2014; auch Rose 2014). Die Erhöhung des Frauenanteils in Naturwissenschaft und Technik ist also

\footnotetext{
${ }^{2}$ Der Begriff der Arbeit ist seit jeher Gegenstand der Geschlechterforschung und bedarf einer Differenzierung, etwa in Erwerbsarbeit und (unbezahlte) Reproduktionsarbeit. Zur Geschichte und feministischen Kritik an der von Marx herrührenden Unterscheidung von Produktions- und Reproduktionsarbeit vgl. weiterführend etwa Haug (1999) und Notz (2011).

${ }^{3}$ Wenn hier und im Folgenden von ,männer- und frauendominierten ' Berufsfeldern die Rede ist, meint dies ausschließlich das quantitative Geschlechterverhältnis.
} 
mit der Hoffnung auf eine Umwälzung geschlechtsbezogener Machtverhältnisse verbunden, die Erhöhung des Männeranteils in sozial(pädagogisch)en Berufen nicht.

Dass Frauen seltener ein technisches Studium beginnen, wird insbesondere auf die Wirkung von Stereotypen (Makarova/Aeschlimann/Herzog 2016; Schmirl et al. 2012), fachkulturelle Elemente der Sozialisation und das Fehlen von Vorbildern (Wensierski/Langfeld/Puchert 2015; Schüller/Braukmann/Göttert 2016), die geschlechtsspezifische Entwicklung eines fachlichen Selbstkonzeptes (SchiepeTiska/Simm/Schmidtner 2016) und die vergeschlechtlichte Konstruktion von ,Technik ${ }^{4}$ an sich zurückgeführt (Paulitz 2012; Paulitz 2015; Schmeck 2019). Wie die Wege von Frauen in soziale Studiengänge und Berufe entstehen und welche Faktoren die entsprechenden Orientierungsprozesse bei Männern - auch im Ingenieurbereich - beeinflussen, wird dagegen nicht bzw. nur vereinzelt betrachtet (Ganß 2011; Puchert 2017). Und auch die Rolle der sozialen Herkunft im Prozess der Studien(fach)wahl ist in der aktuellen Geschlechterforschung weniger en vogue. Das liegt vor allem an der dominanten poststrukturalistischen und diskurstheoretischen Ausrichtung zeitgenössischer geschlechtertheoretischer Forschungen, die die diskursiven Verknüpfungen von Beruf und Geschlecht erkenntnisreich herausarbeiten, hinter denen aber das Interesse an strukturellen Mechanismen sozialer Ungleichheiten zurücktritt ${ }^{5}$. Ein Ansatz der Geschlechterforschung, der dagegen Beruf und Berufswahlen strukturell rahmt und gleichzeitig die sozialen Konstruktionsprozesse von Geschlecht betrachtet, stammt von Angelika Wetterer und hat besonderes Potential für die Analyse entsprechender Ungleichheitsmechanismen (Wetterer 1995; Wetterer 2002): Wetterer erklärt die vergeschlechtlichte Arbeitsteilung zu dem zentralen Moment der Herstellung von Geschlecht und zeichnet die soziale Konstruktion von Beruf und Geschlecht als gleichzeitige und miteinander verwobene Prozesse nach. Dabei erklärt sie die Vergeschlechtlichung

\footnotetext{
${ }^{4}$ Mit einfachen Anführungszeichen wird in dieser Arbeit eine Distanzierung vom jeweils verwendeten Begriff vorgenommen. Doppelte Anführungszeichen sind wörtlichen Zitaten (aus der Literatur bzw. aus dem empirischen Material) vorbehalten.

${ }^{5}$ Diskurstheoretische Positionen haben in den letzten Jahren der Geschlechterforschung einen besonderen Stellenwert eingenommen: Die Existenz von zwei Geschlechtern als ein Resultat eines primär sprachlichen Konstruktionsprozessen zu fassen, kann mittlerweile als „,nicht mehr hintergehbarer Standard“ (Soiland 2011: 23) bezeichnet werden - Geschlecht überwiegend oder gar ausschließlich als Produkt von Diskursen zu begreifen, bestimmt den geschlechtersoziologischen Diskurs. Die damit verbundene Akzentverschiebung feministischer Ansätze ,,von der Umverteilung zur Anerkennung“ im Sinne eines feministischen (und teilweise einseitigen) Kulturalismus führt etwa nach Nancy Fraser (2009) in der Folge dazu, dass sozialökonomische Kämpfe denen um Anerkennung untergeordnet werden, statt sie zu ergänzen.
} 
und Hierarchisierung von Berufen zum Strukturmoment und begründet damit ihren Ansatz des doing gender while doing work ${ }^{6}$. Welche Bedeutung der sozialen Klasse in diesem Prozess zukommt, spielt bei Wetterers professionssoziologischem Ansatz jedoch keine Rolle - der zudem bislang nicht bzw. nur ungenügend auf Berufswahlen angewandt wurde. Was in der Geschlechterforschung daher fehlt, ist eine Perspektive auf Berufs- und Studien(fach)wahlen, die - wie jene von Wetterer - Handeln und Struktur miteinander verbindet, die nicht nur, geschlechtsatypische" Berufswahlen betrachtet und die zudem die Bedeutung von Klasse bzw. der sozialen Herkunft systematisch berücksichtigt.

\section{Studien(fach)wahlen in der Bildungsforschung}

Denn es lässt sich nicht leugnen, wie immens Bildungswege strukturell gerahmt und durch die soziale Herkunft bestimmt sind. Die Frage nach entsprechenden Selektionsmechanismen hin zum Studium wird in der Bildungsforschung insbesondere seit den 1960er-Jahren gestellt (Bargel 2006): Zu dieser Zeit standen Barrieren in den Schul- und Bildungswegen von Kindern und Jugendlichen im Mittelpunkt sowie die ,männlich geprägte Universität" und die Situation von Arbeiter*innenkindern an Universitäten. Die Diagnosen von Hansgert Peisert (1967) und Ralf Dahrendorf (1966) zur mehrfachen bildungsbezogenen Benachteiligung ,der Landkinder, Arbeiterkinder und der Mädchen, zu denen mit gewissen Einschränkungen als vierte Gruppe katholische Kinder kommen“ (ebd.: 48) prägten maßgeblich den Ruf nach einer sozialen Öffnung des Bildungssystems. ${ }^{7}$ Ziel müsse Chancengleichheit durch ein Bildungssystem jenseits „systematische[r] Bevorzugen oder Benachteiligungen bestimmter Gruppen aufgrund leistungsfremder Merkmale wie Herkunft oder wirtschaftlicher Lage" sein (ebd.: 24). Um diese ,soziale Öffnung“ der Hochschulen herbeizuführen, sollte im Zuge der Bildungsexpansion eine tiefgreifende Reform der öffentlichen Bildungssysteme stattfinden und es wurden etwa Fachhochschulen und neue Universitäten gegründet mit dem Ziel, den Anteil an studierenden Arbeiter*innenkindern und Kindern aus „,bildungsfernen Elternhäusern“ zu erhöhen (Bargel 2006: 1). Zwar kam es so zu einem allgemeinen Anstieg der Bildungsbeteiligung und zu einem gestiegenen Anteil an höheren Bildungsabschlüssen, aber der erhoffte Abbau

\footnotetext{
${ }^{6}$ Kursive Textabschnitte werden in dieser Arbeit erstens bei englischen Fachtermini eingesetzt und zweitens zur inhaltlichen Strukturierung, um also auch andere Fachbegriffe und inhaltlichen Abschnitte hervorzuheben.

${ }^{7}$ Bereits dieser frühe Blick auf Ungleichheiten im Hochschulzugang zeigt, dass das Geschlecht in den entsprechenden Studien der Bildungsforschung nicht ausgeblendet wurde.
} 
sozialer Bildungsungleichheiten blieb aus: Durch die Entwicklungen der Bildungsexpansion stellen zwar nicht mehr formale Abschlüsse die entscheidenden Hürden im Bildungsverlauf dar, aber Selektionsmechanismen wirken nach wie vor und die „Demokratisierung der Bildungsbeteiligung verfeinert[e] (...) die Verschleierungsprinzipien der Reproduktion sozialer Verhältnisse“" (Thiersch 2014: $92 \mathrm{f}.)^{8}$.

Die Betrachtung ebendieser verschleierten Reproduktionsmechanismen sozialer Verhältnisse und der damit verbundenen Ausschlüsse von bestimmten Bildungswegen findet in der Bildungssoziologie entlang von zwei zentralen Erklärungsmustern statt: Rational-Choice-Ansätze im Anschluss an Raymond Boudon (1974) gehen von stärker rationalen Entscheidungen aus, während die reproduktionstheoretische Perspektive im Anschluss an Pierre Bourdieu (1982) das Prozesshafte hinter Bildungsentscheidungen betont, die nach Bourdieuschem Verständnis auf der Grundlage sozialisatorisch inkorporierter und vorreflexiv wirkender Bewertungs- und Handlungsmuster getroffen werden. Und auch wenn beide Perspektiven nicht als unvereinbar gelten (Vester 2006), sondern partiell einander angenähert und miteinander verknüpft werden (Merkel 2015; GrohSamberg/Hertel 2011), kann aufgrund ihrer unterschiedlichen Schwerpunktsetzung und ihrer überwiegend kontrastierenden Rezeption von einem „Paradigmenstreit zwischen Boudon und Bourdieu“ (Kramer/Helsper 2010: 103) gesprochen werden.

Dabei lässt sich mit Kramer und Helsper festhalten, dass das „Erklärungspotential von Bourdieu noch nicht ausgeschöpft ist“" (Kramer/Helsper 2010: 120 f.) und sein Ansatz bietet sich auch besonders an, um die sozialen Logiken von Studien(fach)wahlen zu entschlüsseln: Nach dem Habituskonzept sind Studien(fach)wahlen langfristig angebahnt und sie bilden inkorporierte gesellschaftliche Strukturen ab. Die Wahl eines Studienfachs ist demnach das Ergebnis eines biografisch entstandenen Passungsprozesses „des Habitus zu bestimmten Fächern, Studieninhalten und fächertypischen Vermittlungs- und Lehrformen, Anforderungen des Studiums und zur Fachkultur als Ganzes“ (Bremer/LangeVester 2019: 32 f.). An das Habituskonzept knüpft die Milieuforschung nach Michael Vester und anderen an (2001), die soziale Klassen mit Bourdieu als handelnde Klassen - bzw. Milieus - versteht, die durch ihren gemeinsamen Habitus miteinander verbunden sind. Forschungen, die Studien(fach)wahlen und Studierpraktiken auf Basis des Habitus- und Milieukonzepts betrachten, sind rar (etwa Lange-Vester/Teiwes-Kügler 2004; Schneider 2016; Grunau 2017; Hild 2019) und zeigen auf, dass die Wahl eines Studienfachs eingebettet ist in die

\footnotetext{
${ }^{8} \mathrm{Zu}$ den Folgen der Bildungsexpansion siehe auch weiterführend Hadjar/Becker (2006).
} 
gesamte Lebensführung und Studierende je nach Dispositionen zu bestimmten Studienfächern und Studierpraktiken neigen. Vor diesem Hintergrund lässt sich nachzeichnen, dass das Studium ,....Teil eines Transformationsprozesses [ist], der Dispositionen des Habitus in Positionen sozialer Ordnung umwandelt“" (ebd.). Worauf in den Studien zu Habitus und Studium wiederum nur am Rande ein analytischer Blick gelegt wird, ist die Habitusdimension von Geschlecht. Auch an diese Leerstelle knüpft diese Arbeit an, indem der Habitusansatz mit anschlussfähigen Konzepten der Geschlechterforschung verknüpft wird, um so die habitusspezifischen - und damit vergeschlechtlichten und milieuspezifischen - Muster von Studien(fach)wahlen zu analysieren.

\section{Forschungsfrage}

Auf diesen Grundlagen soll die leitende Forschungsfrage beantwortet werden, wie Studien(fach)wahlen im Kontext von sozialem Milieu und Geschlecht entstehen. Mit dieser leitenden Frage sind noch weitere Fragestellungen verknüpft, denen mit dieser Arbeit nachgegangen werden soll:

- Welche habitusspezifischen Dispositionen prägen die Wahl eines sozial- bzw. ingenieurwissenschaftlichen Studiums, welche Affinitäten und Passungsverhältnisse deuten sich hier an?

- Welche Spuren der Vergeschlechtlichung zeichnen sich in den milieuspezifischen Habitusmustern der Studien(fach)wahl ab?

- Wie sind diese Muster der Studien(fach)wahl eingebettet in die (vergeschlechtlichte) Lebensführung und -planung, insbesondere mit Blick auf die antizipierte Familiengründung und -organisation?

Das Ziel dieser Arbeit verweist damit auf ein besonderes Merkmal des Habituskonzepts: Denn von Seiten der Geschlechterforschung wird auf „das Potenzial“ der Bourdieuschen Perspektive verwiesen, „Analysen von Intersektionalität zu revolutionieren“ - so die Einschätzung von Helma Lutz (Yuval-Davis 2013: 194) .

\footnotetext{
${ }^{9}$ Die Grundannahme der Intersektionalität besagt, dass die Gesellschaft durch mehrere Dimensionen sozialer Ungleichheit geprägt ist und das Geschlecht nur eine davon ist - zugleich fragt sie danach, welche anderen „Kategorien“ (Hagemann-White 2011: $20 \mathrm{f}$.) wann relevant sind und in welchem Verhältnis sie zueinander. Ob Intersektionalität dabei als Theorie, als Konzept oder heuristisches Instrument gefasst werden kann, ist fraglich und umstritten - nach wie vor gilt sie als „Buzzword“ (Davis 2013) der Geschlechterforschung, das gerade durch seine Vagheit und Offenheit besticht. Vergleiche weiterführend zum Ansatz der Intersektionalität auch Winker/Degele (2009); Bührmann (2009); Lutz/Vivar/Supik (2013b); Degele/Winker (2011); Vinz (2011).
} 
Der Habitus ermöglicht es, verschiedene Dimensionen sozialer Ungleichheit als inkorporierte soziale Strukturen zu fassen und sie damit zusammenzudenken. Dieses Potential wird in der vorliegenden Arbeit ausgelotet und das Zusammenspiel von Klasse und Geschlecht im Kontext von Bildungsungleichheit, scharf gestellt‘.

\section{Vorgehen dieser Arbeit}

Studien(fach)wahlen werden zunächst (Kapitel 2) aus Perspektive der quantitativen Bildungsforschung betrachtet: Es wird gezeigt, wie sich die deutsche Studierendenschaft aktuell zusammensetzt, welcher Selektionsprozess dem Eintritt in die Hochschule vorausgeht und welche Studienmotive mit der Aufnahme eines Studiums verbunden sind. Schon hier wird deutlich werden, dass der Eintritt in die Hochschule maßgeblich durch die soziale Herkunft geprägt ist, während bei der Fachwahl soziale Herkunft und soziales Geschlecht eine entscheidende Rolle spielen. Der Ansatz der Motivforschung ermöglicht allerdings nicht die Bearbeitung der Frage, wie die entsprechenden Motive zu erklären sind und warum das Geschlecht die Studienfachwahl derart beeinflusst.

Dazu bedarf es einer tiefgehenden Ursachenerkundung (Kapitel 3). Diese beginnt mit einem Blick auf die Perspektiven der Geschlechterforschung auf Studien(fach)wahlen (Abschnitt 3.1): Die Ansätze der Geschlechterforschung ${ }^{10}$ fokussieren sich schwerpunktmäßig auf die Wege von Frauen in die Ingenieurfächer und -berufe, beziehen aber auch die Fachwahl von Männern und andere Dimensionen sozialer Ungleichheit wie die soziale Herkunft ein - dies allerdings nur punktuell und häufig ohne die Berücksichtigung eines differenzierten Verständnisses von sozialer Klasse. Um neben dem Geschlecht auch die soziale Herkunft systematisch zu betrachten, benötigt es daher einen erneuten Rückgriff auf den Stand der Bildungsforschung und insbesondere ihre sozialwissenschaftlichen Erkenntnisse zur Entstehung von Studien(fach)wahlen (Abschnitt 3.3). Im Anschluss an Bourdieu und die anknüpfende Milieuforschung lässt sich aufzeigen, wie sich Studien(fach)wahlen im Kontext milieuspezifischer Passungsprozesse greifen lassen können. In der Verknüpfung von geschlechter- und

\footnotetext{
${ }^{10}$ Die Ansätze der Geschlechterforschung zeichnen sich durch ihre Interdisziplinarität aus, die sich auch in Abschnitt 3.1 wiederspiegelt, in dem allerdings ein geschlechtersoziologischer Fokus gelegt wird. Um dieser Vielfalt zugleich gerecht zu werden und sie für die Betrachtung von Studien(fach)wahlen zu nutzen, werden Ansätze der Biografieforschung, der Sozialpsychologie bzw. oder der Professionssoziologie, die sich explizit mit der Konstitution von Geschlecht befassen, den Perspektiven der Geschlechterforschung zugeordnet. Deutlich wird auch, dass eine scharfe Trennung von Geschlechter- und Bildungsforschung hier nicht immer möglich ist - so fließen auch Elemente der psychologischen und fachdidaktischen Bildungsforschung, die die Dimension von Geschlecht in der Entstehung von Studien(fach)wahlen fokussieren, in den Stand der Geschlechterforschung mit ein.
} 
bildungstheoretischen Perspektiven können Studien(fach)wahlen so als vergeschlechtlichte und klassenspezifische Passungsprozesse verstanden werden, die zum strukturellen (Selbst-)Ausschluss bestimmter Habitus aus prestigeträchtigen beruflichen Positionen führen.

Um diese Passungsprozesse analytisch zu greifen, eignen sich das Habituskonzept und die daran anschließende Milieuforschung nach Vester, die mit der Dimension von Geschlecht verknüpft werden (Kapitel 4). Im Ergebnis werden Studien(fach)wahlen als Ausdruck vergeschlechtlichter und vergeschlechtlichender Milieuhabitus gefasst.

Diese theoretischen Grundlagen werden schließlich empirisch angewendet, um den sozialen Logiken von Studien(fach)wahlen im Kontext von sozialem Milieu und Geschlecht nachzuspüren. Dazu wird mit der Habitushermeneutik ein methodologischer Zugang zur Analyse von Studien(fach)wahlen gewählt, der die Dimensionen von Geschlecht und Milieu gleichermaßen umfasst (Kapitel 5). Es folgt der das empirische Herzstück dieser Arbeit: Anhand von acht Vergleichsfällen aus der Sozialen Arbeit und dem Bauingenieurwesen an einer Hochschule für Angewandte Wissenschaften werden habitusspezifische Wege in ein Studium analysiert und entsprechende Milieuhypothesen aufgestellt (Kapitel 6). Anschließend werden diese Wege als Ausdruck spezifischer sozialer Positionen auf einer übergeordneten Ebene von milieuspezifischen und vergeschlechtlichen Mustern der Studien(fach)wahl herausgearbeitet (Kapitel 7). Schließlich werden die Ergebnisse zusammengefasst und diskutiert, in die aktuellen Diskurse von Geschlechter- und Bildungsforschung eingeordnet und für weitere Ansätze von Praxis und Forschung aufbereitet (Kapitel 8). 
Open Access Dieses Kapitel wird unter der Creative Commons Namensnennung 4.0 International Lizenz (http://creativecommons.org/licenses/by/4.0/deed.de) veröffentlicht, welche die Nutzung, Vervielfältigung, Bearbeitung, Verbreitung und Wiedergabe in jeglichem Medium und Format erlaubt, sofern Sie den/die ursprünglichen Autor(en) und die Quelle ordnungsgemäß nennen, einen Link zur Creative Commons Lizenz beifügen und angeben, ob Änderungen vorgenommen wurden.

Die in diesem Kapitel enthaltenen Bilder und sonstiges Drittmaterial unterliegen ebenfalls der genannten Creative Commons Lizenz, sofern sich aus der Abbildungslegende nichts anderes ergibt. Sofern das betreffende Material nicht unter der genannten Creative Commons Lizenz steht und die betreffende Handlung nicht nach gesetzlichen Vorschriften erlaubt ist, ist für die oben aufgeführten Weiterverwendungen des Materials die Einwilligung des jeweiligen Rechteinhabers einzuholen.

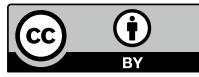

